\title{
Diagnostic potential of chromogenic substrates for rapid detection of bacte- rial enzymatic activity in health and disease associated periodontal plaques
}

\author{
S. A. SYFi, F. A. Gusberit, W. J. Loescife and N. P. LANG
}

Dental Research Institute, School of Dentistry. The University of Michigan, Ann Arbor, USA and Laboratory of Oral Microbiology, School of Dental Medicine, University of Berne. Switzerland

\section{Introduction}

Microbiological investigations have shown that distinct microbial populations are associated with bealthy or diseased periodontal tissues (Loesche 1982). Several microscopic and microbiological approaches have been attempted in recent years to diagnose and monitor periodontal conditions in humans. These include examination of morphological types and motility of bacteria by darkfield or phase-contrast microscopy and microbiological culturing of subgingival plaque to determine the composition of the cultivable flora.

These methods may, however, not be suitable for routine use as they are time consuming, are limited to the analysis of few plaque samples, and require expert handling from the time of sampling through the analysis of the plaque specimens.

Studies from several microbiological laboratories demonstrate that some periodontopathic bacteria may also be characterized by their enzymatic activities (Dzink, Smith \& Socransky 1984, Hofstad 1980 , Laughon, Syed \& Loesche 1982a, Slots 1981. Syed et al. 1984, Syed et al. 1984). The detection of selected bacterial enzymatic reactions directly in plaques associated with periodontal diseases may therefore be an casy and efficient method of diagnosis.

The present study evaluated the potential of a rapid test system for the detection of specific bacterial enzymatic activities in some periodontally defined populations.

\section{Materials and Methods}

Selection of substrates and test procedure The following $\beta$-naphtylamide derivatives (Sigma) were chosen for the development of the rapid test system: $N$ - $\alpha$-benzoyl-DLarginine (BANA) (Try), L-pyrrolidine (Pyr), L-phenylalanine (Phe), L-leucine (Leu). Lvaline (Val), L-serine (Ser), L-arginine (Atg), and L-alanine (Ala).

The selection of these substrates was based on the enzymatic reactions of specific bacterial species belonging to the genera: Bacteroides. Treponema, Actinomyces, Capnocytophaga, Fusohacteriam, Veillonella. and Sclenomonas. In addition, species of these genera were tested at decreasing concentrations to ascertain the efficiency of the system for the detection of the correspond ing enzymatic reactions.

With the exception of BANA each subs. trate was prepared by suspending $0.016 \mathrm{~g} \mathrm{o}$ ! the powder in $1 \mathrm{ml}$ dimethylsulfoxide, fol- 
lowed by the addition of $20 \mathrm{ml}$ of glass distilled water. The substrates were dispensed in aliquots of $100 \mu \mathrm{l}$ in 96 wells microtiter plates and kept frozen at $-20^{\circ} \mathrm{C}$. until used.

BANA was prepared as previously described (Laughon, Syed \& Loesche 1982b). Addition of tris-hydrochloride buffer and dispensing in the microtiter plates was performed just before use.

Single samples of bacterial plaque were collected with a sterile curette and suspended by vigorous agitation in small presterilized screw-capped glass vials containing $1 \mathrm{ml}$ of $0.01 \mathrm{M}$ HEPES-buffer ( $\mathrm{pH} 7.2$ ) with glass-beads. Care was taken to collect as much plaque as possible and up to the deepest part of periodontal sulci or pockets. The vials containing the plaques were vortexed for 5-10 seconds and aliquots of approximately $100 \mu \mathrm{l}$ added to each substrate in the microtiter plates previously brought to room temperature. The plates were then covered with Para-Film and incubated overnight at $35^{\circ} \mathrm{C}$ in a humidified container.

After incubation, $100 \mu \mathrm{l}$ of a color developing reagent (acidified solution of p-dime(hylaminocinnamaldehyde) was added to each well, and the results recorded within five minutes. The development of colors from dark red to purple was considered positive. Yellow to orange colors were considered negative reactions. Appropriate controls were always included for each exseriment.

\section{"atient populations}

The first population consisted of 16 young idults who volunteered for an experimental ingivitis study. Two weeks prior to the ommencement of the study (day -14), ndividual plaque samples from eight preelected sites were collected in each subject. A second series of samples was obtained rom the same sites at the termination of the study (day 21). Prior to the beginning of the gingivitis study (days -14 to 0 ), all subjects received full mouth-scaling and oral hygiene instructions. At the beginning of the study (day 0 ), they were randomly divided into two groups of eight subjects. During a 21 day period of no mechanical oral hygiene, each group rinsed daily with a different antimicrobial solution containing a quaternary ammonium compound. Plaque (PI) and Gingivitis Index (GI) scores were recorded at day 0 and 21 . No pockets deeper than $4 \mathrm{~mm}$ were present at the selected sampling sites in these subjects.

The second group consisted of 21 patients in the maintenance phase (recall patients). For each patient, bacterial plaque was collected from the 8 deepest periodontal sites. Pocket probing depth was measured after sample coliection and the results of the enzymatic reactions divided into two groups according to the pocket probing depths values.

The third group included 7 patients with untreated periodontitis. Selection of sites, plaque collection, and pocket probing depth measurements were performed as for the recall group.

\section{Results and Discussion}

The substrates selected covered the enzymatic reactions of a broad spectrum of bacterial species associated with gingivitis and periodontitis. The minimal number of bacteria required for positive enzymatic reactions varied with the bacterial species and substrates. Generally, a bacterial density from $10^{5}$ to $10^{9} / \mathrm{ml}$ was sufficient for positive enzymatic reactions.

The rapid test system enabled the detection of bacterial enzymatic activities in plaques taken from individual tooth sites in populations with different periodontal conditions (Table 1). The experimental gingivitis group at day -14 showed little or no 


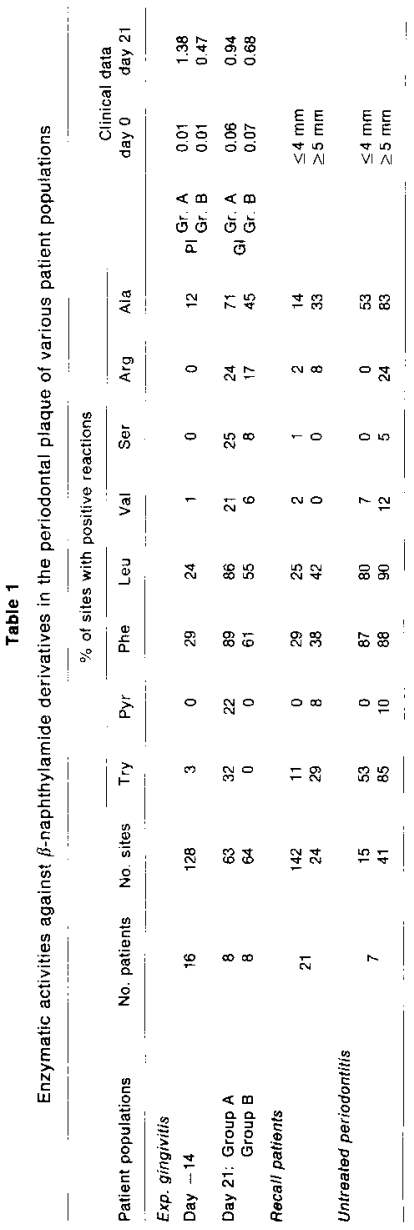

enzymatic activities except for Phe and Leu, reflecting the absence or minimal levels of disease in this population of young adults. After $2 l$ days of no oral hygiene, an increase in the number of sites with positive enzymatic reactions was observed predominantly in group A, i.e. the group that utilized a clinically less effective antimicrobial mouthrinse solution as revealed by $\mathrm{PI}$ and GI scores. Accordingly, in group $B$ the number of sites with positive reactions for the various enzymes was lower. None of the sites in this group showed positive reactions for Try or Pyr.

From these results, it may be concluded that the onset of gingivitis would correlate with the increase of the enzymatic activities in the developing plaque. In the population of patients in maintenance, various degrees of enzymatic reactions were observed. Higher proportions of positive tests were obtained from the samples of the deeper pockets than from the shallow ones. This would indicate the presence of a greater bacterial mass in deep periodontal sites.

The comparison of the data between this group of patients and the younger individuals of the experimental gingivitis group showed no major differences of the enzymatic activities for the shallow sites except an increase of Try activity in the maintenance group. This may indicate the possible occurrence of periodontopathic bacteria in? a few sites during maintenance.

In the untreated periodontitis patients. similar trends in the enzymatic reactions were found between shallow and deep pockets as in patients in maintenance. Plaqu: collected from this population reveales: higher proportions of sites with enzymati activities than in the maintenance group o: in the gingivitis group at day -14 . Ir untreated periodontitis, where plaque cal usually be collected in copious amounts enzymatic reactions for Leu or Phe wer positive irrespective of pocket depth, where- 
as enzymes for other substrates were more discriminative for different levels of disease severity as expressed by pocket depth.

Enzymatic reactions for Try, Pyr, and Ala could therefore be of more diagnostic value for monitoring disease severity as evidenced by the comparison of values between the population groups and the sites with different pocket depths.

In summary, the use of chromogenic substrates for rapid detection of bacterial enzymatic activities could be a useful method for diagnosing and monitoring patients with different periodontal conditions. This system would determine the density of the microflora at a given tooth site and discriminate between healthy and disease associated plaques by revealing the presence of enzymatic reactions specific for periodontopathic bacteria. Longitudinal evaluations are required for the assessment of its possible use as a diagnostic tool for disease activity. Studies for further improvement of this test system are in progress.

\section{Acknowledgements}

This research was supported by NIDR Grant No. DE 02731 and Swiss National Foundation Grant No, 3.953-82.

\section{References}

Jzink, J. L.. Smith, C. \& Socransky, S. S. 1984. Semiautomated technique for identification of subgingival isolates. Journal of Clinical Microbiology 19: 599 605.

Hofstad, T. 1980. Evaluation of the API-ZYM System for identification of Bacteroides and Fusobacterium spccies. Medical Microbiology and immunology 168: 173-177.

Laughon, B. E., Syed, S. A. \& Loesche, W. J. 1982a. API ZYM system for identification of Bacteroides spp., Capnocytophagu spp. and spirochetes of oral origin. Journal of Clinical Microbiology 15: 97-.102.

Laughon, B. F., Syed, S. A. \& Loesche, W. J. 1982b. Rapid identification of Bacteroides gingivalis. Journal of Clinical Microbiology 15 : $345 \cdot 346$.

Loesche. W. J. 1982. The bacterial etiology of dental decay and periodontal disease: The specific plaque hypothesis. In: Clinical Dentistry. ed. Clark, J. W., Vol. 2, ch. 1A. pp. 1-13. Philadelphia: Harper and Row, Publishers.

Stots, J. 1981. Enzymatic characierization of some oral and non-oral gram-negative bacterja with the API ZYM system. Journal of Climical Microbiology: 14: $288 \cdot 294$

Syed, S. A., Loesche, W. J., Gusberti, F. A. \& Lang. N. P. 1984. Rapid characterization of periodontopathic bacteria by chromogenic substrates. Journal of Dental Research 63: Special Issue. Abstracts p. 263. Abstr. No. 834.

Syed, S. A., Loesche, W. J., Gusberti, F. A., Lang, N. P. \& Pearson, C. 1984. Efficiency of rapID Ana System for identification of oral and non-oral bacteria. Abstracts of the annual meeting. p. 262. Abstract No. C-155. American Socicly for Microbiology.

Address:

Dental Research Institute

School of Dentistry

The University of Michigan

Anm Arbor. Michigan 48709

U.S.A 
This document is a scanned copy of a printed document. No warranty is given about the accuracy of the copy. Users should refer to the original published version of the material. 